\title{
Synthesis and Characterization of Compounds Based on Carbazole and Sulfone Groups
}

huixian jia ( $\square$ huixian0425@163.com )

Research Institute of Chemical Defense https://orcid.org/0000-0001-8520-5467

\section{Chenghong Feng}

Beijing Normal University

\section{Wanfei Han}

Research Institute of Chemical Defense

\section{Yan Xue}

Research Institute of Chemical Defense

Yanren Jin

Research Institute of Chemical Defense

Dongfeng Li

Changchun University of Technology

\section{Research Article}

Keywords: Luminescence, Carbazole, Sulfone, Charge transfer

Posted Date: July 15th, 2021

DOl: https://doi.org/10.21203/rs.3.rs-698108/v1

License: (c) (i) This work is licensed under a Creative Commons Attribution 4.0 International License. Read Full License

Version of Record: A version of this preprint was published at Journal of Fluorescence on November 12th, 2021. See the published version at https://doi.org/10.1007/s10895-021-02844-8. 


\title{
Synthesis and characterization of compounds based on carbazole and
}

\author{
sulfone groups \\ Huixian Jia ${ }^{\mathrm{a}, \mathrm{b} *}$, Chenghong Feng ${ }^{\mathrm{c}}$,Wanfei Han ${ }^{\mathrm{a}}$,Yan Xue ${ }^{\mathrm{a}}$,Yanren $\mathrm{Jin}^{\mathrm{a}}$,Dongfeng $\mathrm{Li}^{\mathrm{b}}$, \\ ${ }^{a}$ Shanxi Xinhua Chemical Defense Equipment Research Institute Co., Ltd.., Taiyuan 030008, China \\ ${ }^{b}$ School of Chemistry and Life Science, Changchun University of Technology, Changchun 130012, \\ China \\ ${ }^{c}$ State Key Laboratory of Water Environment Simulation, School of Environment, Beijing Normal \\ University, Beijing 100875, China \\ *Corresponding authors Tel/Fax: +86 18435165116, \\ E-mailaddresses: huixian0425@163.com;
}

\begin{abstract}
Two compounds containing carbazole and sulfone groups with different alkyl chain lengths have been designed and synthesized. The sulfone group has strong absorption characteristics and the alkoxy chain and carbazole group are electron-rich, forming $\mathrm{D}-\delta$-A-type symmetrical molecules. The molecules have the characteristics of charge transfer and high thermal stability, and the molecules stack to form a layered staggered stack, reducing the intermolecular $\pi-\pi$ interactions. The target compounds also exhibit strong ultraviolet-fluorescent emission in the solid state at room temperature, and they are expected to be good luminescent materials.
\end{abstract}

Keywords: Luminescence; Carbazole; Sulfone; Charge transfer

\section{Introduction}

Carbazole has a stable planar conjugated rigid structure, the structure of nitrogen atoms in the soliton is a fused ring structure of the compound, while the carbazole group is a group with excellent photoelectric properties. It is not only good thermal stability, but also a multi-site chemical reaction. Carbazole designed in the photoelectric functional materials, not only to ensure that it has a good photoelectric properties, but also expand the researchers on the design of the compound ideas. The current research and use of carbazole groups is introduced into the main chain by conjugation, and the carbazole is introduced into the polymer chain in a different polymerization manner to form a polymer material. The combination of carbazole in the alkyl chain to the target compound is relatively scarce. For example: Li G, Zhao J, Zhang D, et al[1]. Synthesize asymmetric blue carbides containing carbazole and 
sulfone structures. The target compound forms a planar conjugated structure with carbazole and benzene sulfone. Qisheng, zhang and JieLi et al[2]. designed and synthesized two compounds containing both carbazole and sulfone structure, but with dendritic compounds at the ends. Bingjia Xu and Haozhong Wu et al[3]. Designed and synthesized two butterfly-type compounds containing thiophene and sulfone Plane height conjugate structure. At the same time, the sulfone structure has a strong electron-withdrawing effect, which contains both carbazole and sulfone structure, and contains less flexible alkyl chain groups. This non-planar, non-highly conjugated compound is less studied. Because the alkyl chain linked carbazole groups have the characteristics of electrons[4], sulfone has a strong absorption characteristics, carbazole itself has the photoelectric properties and charge transport properties, making such materials in the field of optoelectronic materials has great potential. Mean while,the solubility is a very important condition in organic luminescent materials. So introduction alkyl chains in compounds can effectively improve the solubility and also promote high quality film deposition. The stability and lifetime of optoelectronic devices lies in the thermal stability of the compounds. Carbazole derivatives are a class of strong blue light emission, high Tg temperature and thermal stability effects of the compound, making them attractive OLED design candidates.

Current thin-film UV-emitting devices radiate not only UV light but also visible light, so their structure needs to be modified to make the emission wavelength in the UV emission region. These UV organic light-emitting devices (OLEDs) exhibit a primary electroluminescence (EL) peak at approximately $400 \mathrm{~nm}$, and thus they are attractive UV OLEDs[5-7].

In this study, we designed and synthesized two carbazole derivatives with UV luminescence and high stability. We found that the target compounds possess a special $\mathrm{W}$-shaped structure, which weakens the $\pi-\pi$ stacking. The band gap of the compounds is $3.44 \mathrm{eV}$. We found that the $\mathrm{W}$-shaped molecules show strong and tunable light emission in solution and as a solid-state film, and the wavelength of 6 light is in the long-wave UV region. These long-wave UV organic electroluminescent materials have a wide range of potential applications.

\section{Experimental}

\section{Materials and Methods}

\section{Chemicals}

1,4-Dibromobutane and 1,2-dibromoethane were purchased from Bailing 
Technology Co., Ltd. They were used without further purification. Carbazole was purchased from the Tianjin Guangfu Fine Chemical Research Institute (AR). The solvents were purchased from the reagent company. Petroleum ether, acetonitrile and n-heptane were purchased from Tianjin Guangfu Technology Development Co., Ltd.(AR).Ethyl acetate, ethanol, chloroform, and dichloromethane were purchased from Beijing Chemical Plant Co., Ltd.(AR).The solvents were used in the experiments without further purification.

\section{Instrumentation}

The ${ }^{1} \mathrm{H}$ and ${ }^{13} \mathrm{C}$ NMR spectra were recorded with an AV-400 spectrometer (Bruker, Switzerland) at $400 \mathrm{MHz}$. The solvent was deuterated reagent and tetramethylsilane was used as the internal standard. Mass spectrometry was performed with a VG ZAB-HS spectrometer(VG,Britain). The infrared (IR) spectra were recorded with an IR spectrometer(FT-IR, Spectrum One type, Perkin Elmer, USA). The UV-visible (vis) spectra were recorded with a spectrophotometer (model 2550, Shimadzu Corporation, Japan). The fluorescence spectra of the target compounds were recorded with a RF-5301PC fluorescence spectrometer (Shimadzu Corporation,Japan). Elemental analysis was performed with a Vario EL III CHNOS elemental analyzer(Elementar,Germany). The fluorescence quantum yield was determined with a Fluorolog 3-TSCPC fluorescence spectrometer (Horiba Jobin Yvon,USA). The thermal decomposition temperature was determined with a TGA Q500 thermogravimetric analyzer (Perkin Elmer, USA) under $\mathrm{N}_{2}$ flow at a heating rate of $5{ }^{\circ} \mathrm{C}$. Cyclic voltammetry was performed with a CHI-852C electrochemical workstation (Chenhua Instruments,China). It was used to determine the oxidation-reduction potential of the target compounds. Tetrabutyl ammonium bromide was used as the electrolyte. The counter and working electrodes were Pt wire and a Pt disk, respectively, and the reference electrode was $\mathrm{Ag} / \mathrm{AgCl}$. The target compound was dissolved in dichloromethane $\left(0.1 \mathrm{~mol} \mathrm{~L}^{-1}\right)$. Single crystal X-ray diffraction (XRD) of compounds 3-2 and 2-2 was performed with a Bruker D8 ADVANCE single-crystal XRD diffractometer(Bruker,Germany). The selected single crystal sizes were $0.38 \mathrm{~mm} \times 0.19 \mathrm{~mm} \times 0.15 \mathrm{~mm}$. All of the calculations were performed with the SHELXS-97 and OLEX2 programs.

\section{Synthesis and crystal structures}




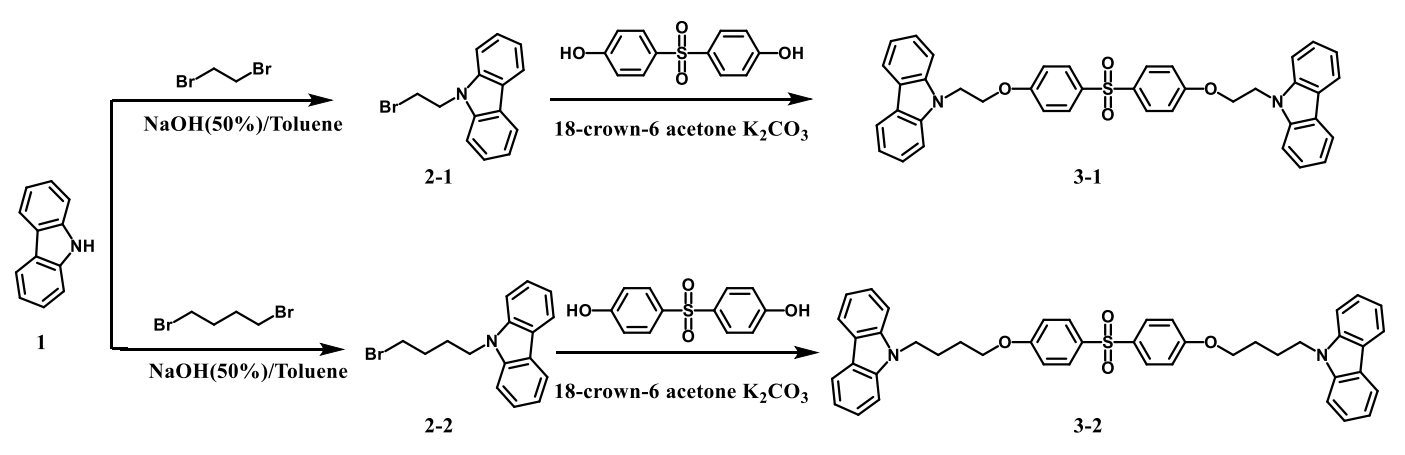

Scheme 1. Synthetic route of the target compounds.

The synthetic procedures for target compounds 3-1 and 3-2 are summarized in Scheme 1. 9-(2-Bromoethyl)-9H-carbazole 2-1 and 9-(4-bromobutyl)-9H-carbazole 2-2 were prepared from carbazole with excess 1,2-dibromoethane and 1,4-dibromobutane, respectively. ${ }^{[8-12]}$ The reaction mixture was slowly heated to $45{ }^{\circ} \mathrm{C}$ and refluxed overnight. The target compounds 3-1 and 3-2 were obtained by the Williamson reaction in acetone and 18-crown-6. Compound 3-2 was obtained as white crystals and 3-1 was a white solid and their yields were $65 \%$. The structures of the target compounds were characterized by ${ }^{1} \mathrm{H}$ and ${ }^{13} \mathrm{NMR}$ spectroscopy, mass spectroscopy, Fourier transform IR (FT-IR) spectroscopy, and elemental analysis.

Compounds 2-2 and 3-2 were obtained by slow evaporation in absolute ethanol/acetone and acetone/ $n$-hexane at room temperature, respectively.[8-12] Crystals suitable for XRD were mounted on a glass fiber for determination, and the data was collected by a Bruker D8 ADVANCE diffractometer with graphite monochromatic $\mathrm{Cu} \mathrm{K \alpha}$ radiation at $296 \mathrm{~K}$. The single crystal of compound $\mathbf{2 - 2}$ is shown in Figure 1 [13]. The analytical software was SAINT v8.37A (Bruker, 2015), SHELXL (Sheldrick, 2015), and Olex2 (Dolomanov). From the single-crystal XRD data,the compound is monoclinic. The stacking of 3-2 is shown in Figure 2 and Figure 3, which shows that 3-2 is W-shaped. From Figure S10 (Supporting Information (SI)), the stacking of the single crystals is a layered interlaced stack. The distance between the sulfur atoms is $7.613 \AA$. The angle between the sulfur atom and the two benzene rings is $105.35^{\circ}$, and the angle between the carbazole and the methylene group is $103.59^{\circ}$. The target molecule is $\mathrm{W}$-shaped. The $\mathrm{W}$ shape reduces the $\pi-\pi$ interaction between molecules. When the distance between the conjugate planes of organic light-emitting molecules is less than $3.5 \AA$, the molecules are likely to accumulate. In contrast, when the distance between the molecules is greater than $7.0 \AA$, the interaction between the molecules is weak and the aggregation effect will be effectively prohibited, and high efficiency of the corresponding light is also 
guaranteed. The distance between the molecules is $7.613 \AA$, so the molecular aggregation effect is inhibited and the luminous efficiency improves. The crystal data is shown in Table S1 in the SI. This interleaved stacking method can reduce fluorescence quenching.

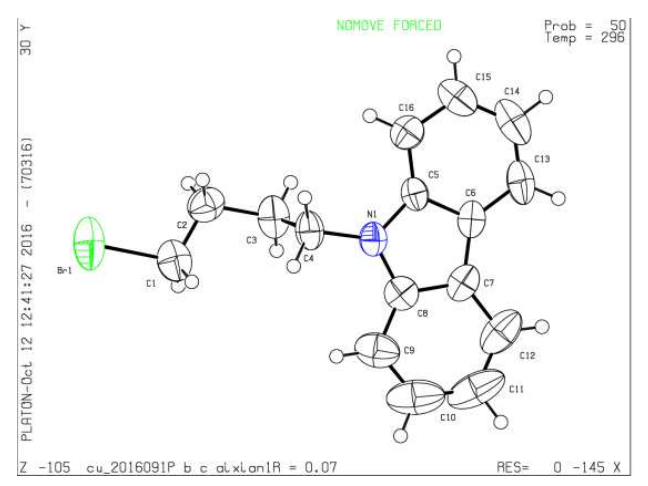

Fig. 1. 9-(4-Bromobutyl)-9H-carbazole molecule.

Table 1. 9-(4-Bromobutyl)-9H-carbazole crystal data.

\begin{tabular}{ll}
\hline Crystal data & \\
\hline Chemical formula & $\mathrm{C}_{16} \mathrm{H}_{16} \mathrm{BrN}$ \\
$M_{\mathrm{r}}$ & 302.20 \\
Crystal system, space group & Orthorhombic, Pbca \\
Temperature (K) & 296 \\
$a, b, c(\AA)$ & $16.0870(4), 7.6967(2), 22.6562(5)$ \\
$V\left(\AA^{3}\right)$ & $2805.22(12)$ \\
$Z$ & 8 \\
Radiation type & Cu Ka \\
m (mm $\left.{ }^{-1}\right)$ & 3.83 \\
Crystal size (mm) & $0.38 \times 0.19 \times 0.15 \mathrm{~mm}$ \\
Data collection & \\
Diffractometer & Bruker P4 \\
Absorption correction & diffractometer \\
Multi-scan \\
independent and & $S A D A B S 2014 / 5$ (Bruker,2014/5) was used for absorption \\
observed $[I>2 \mathrm{~s}(I)]$ & correction. wR2(int) was 0.1658 before and 0.0900 after \\
& correction. The Ratio of minimum to maximum \\
& transmission is 0.3645. The $1 / 2$ correction factor is 0.00150. \\
& $0.275,0.754$ \\
& $19743,2756,2071$ \\
& \\
&
\end{tabular}




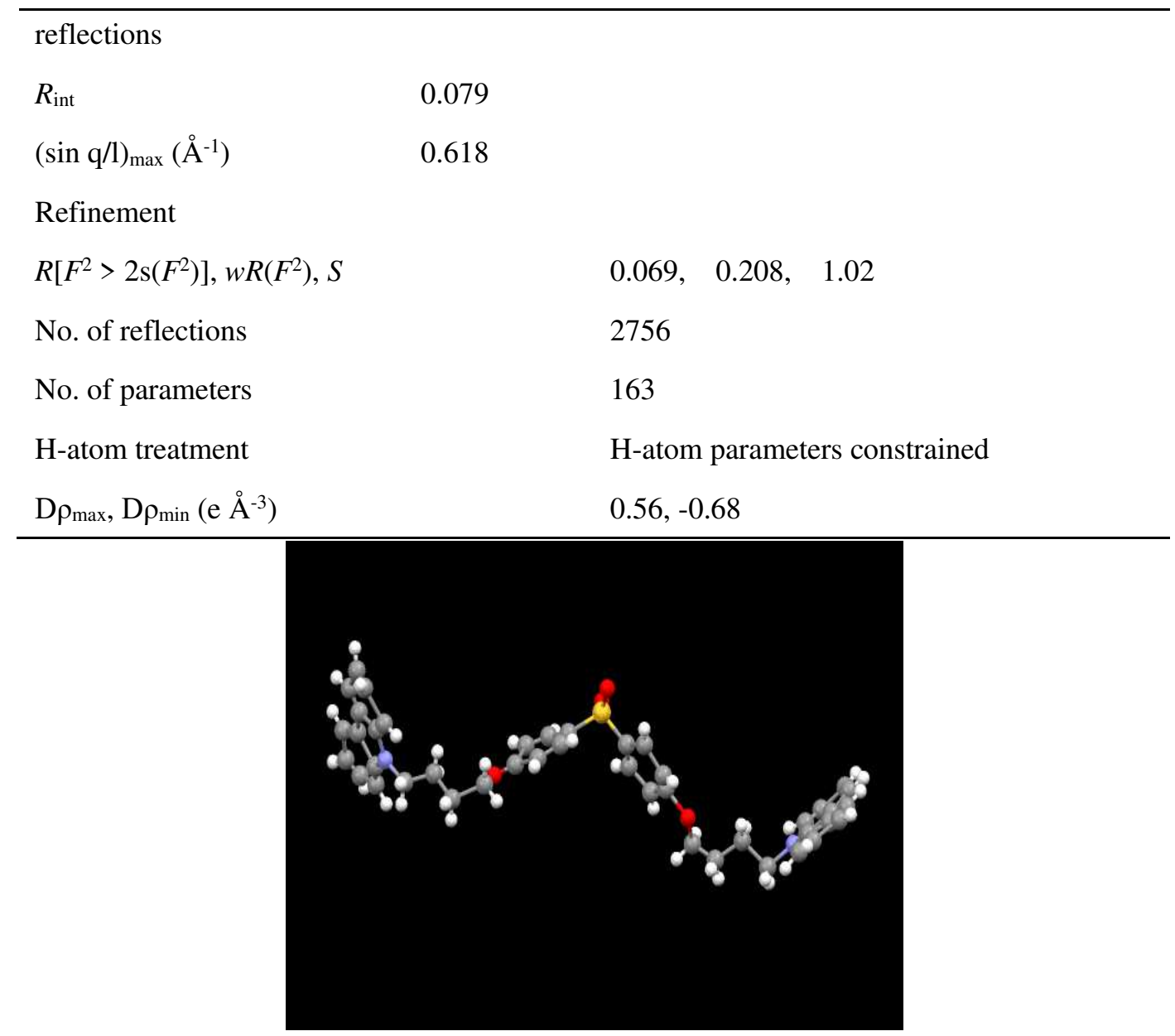

Fig. 2. 9,9'-((Sulfonylbis(4,1-phenylene))bis(oxy))bis(butane-4,1-diyl))bis(9H-carbazole) molecule.
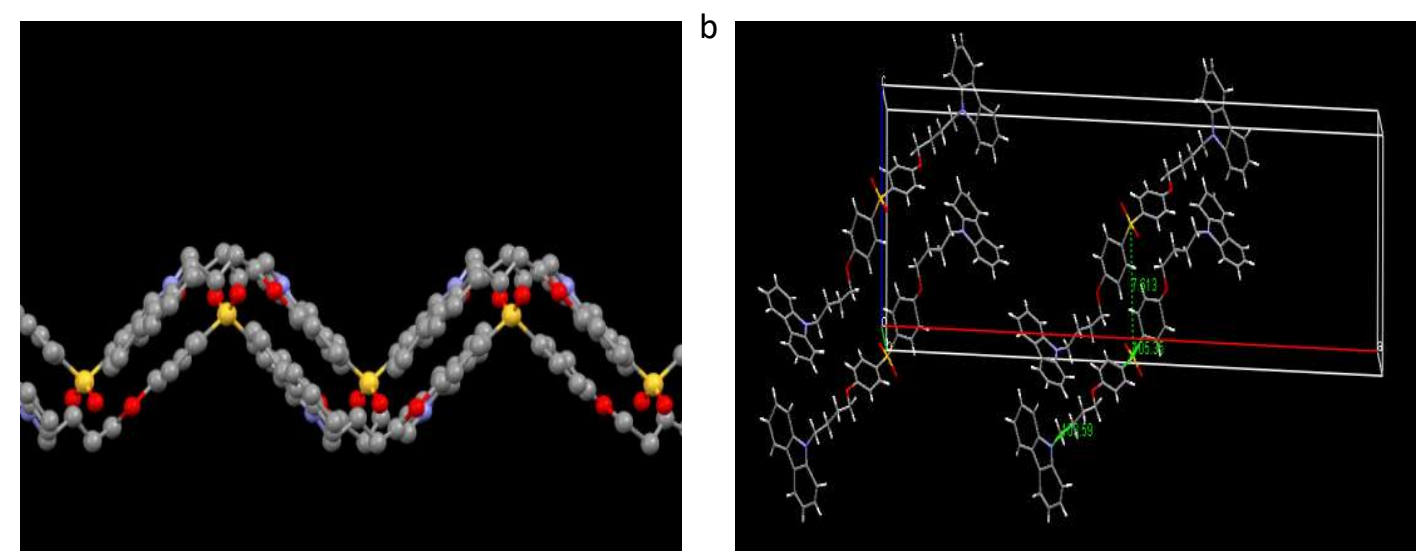

Fig. 3. a) Stacking of compound 3-2.b) Single crystal angles and S-S distance of compound 3-2.

\section{Synthesis of 9-(2-bromoethyl)-9H-carbazole (2-1)}

Carbazole (1.5 g, $0.0089 \mathrm{mmol})$, 1,2-dibromoethane $(5 \mathrm{~mL})$, anhydrous toluene $(6 \mathrm{~mL})$, and Tetrabutylammonium Bromide(TBAB $)(0.76 \mathrm{~g})$ were dissolved in $4.4 \mathrm{~mL}$ $50 \% \mathrm{NaOH}$ solution in a $100 \mathrm{~mL}$ round bottom flask, The reaction temperature was set to $45{ }^{\circ} \mathrm{C}$ and the reaction was performed for $24 \mathrm{~h}$. The reaction was then stopped 
and the mixture was allowed to cool to room temperature. The product was poured into $60 \mathrm{~mL}$ of distilled water and the product was extracted twice with $15 \mathrm{~mL}$ dichloromethane. The organic layer was washed twice with $30 \mathrm{~mL}$ of water, dried overnight, suction filtered, and solvent dried. The crude product was an amber solid, which was purified with boiling $n$-heptane to remove excess 1,2-dibromoethane and a white solid was obtained. The white solid was separated by column chromatography (PE:EA $=10: 1, \mathrm{PE}$ is Petroleum ether and EA is Ethyl acetate) to give white needle-like crystals. The yield was $40 \%$ [8-10].

${ }^{1} \mathrm{H} \mathrm{NMR}\left(\mathrm{CDC}_{1}\right): \delta(\mathrm{ppm})=8.10(\mathrm{~d}, 2 \mathrm{H}, J=7.6 \mathrm{~Hz}), 7.48(\mathrm{~m}, 2 \mathrm{H}, J=7.6 \mathrm{~Hz})$, $7.27(\mathrm{~m}, 2 \mathrm{H}, J=7.6 \mathrm{~Hz}), 4.72(\mathrm{t}, 2 \mathrm{H}, J=7.6 \mathrm{~Hz}), 3.68(\mathrm{~m}, 2 \mathrm{H}, J=7.6 \mathrm{~Hz}) .{ }^{13} \mathrm{C} \mathrm{NMR}$ $\left(\mathrm{CDC}_{3}\right): \delta(\mathrm{ppm})=28.5,45.1,108.8,120.0,120.7,123.6,126.4,140.4$.

\section{Synthesis of 9-(4-bromobutyl)-9H-carbazole (2-2)}

Carbazole (1.5 g, $0.0089 \mathrm{mmol})$, 1,4-dibromobutane (6 mL), anhydrous toluene $(6 \mathrm{~mL})$, and TBAB $(0.076 \mathrm{~g})$ were dissolved in $4.4 \mathrm{~mL} \mathrm{50 \%} \mathrm{NaOH}$ solution in a 100 $\mathrm{mL}$ round bottom flask and protected with nitrogen. The reaction was performed at $45{ }^{\circ} \mathrm{C}$ for $24 \mathrm{~h}$. The reaction was then stopped and the mixture was allowed to cool to room temperature. The product was poured into $60 \mathrm{~mL}$ of distilled water. The product was extracted twice with $15 \mathrm{~mL}$ dichloromethane, and the organic layer was washed twice with $30 \mathrm{~mL}$ of water. The crude product was purified by column chromatography to give a white solid, which was purified by boiling $n$-heptane to remove excess 1,4-dibromobutane and a white solid was obtained. The white solid was separated by column chromatography $(\mathrm{PE}: \mathrm{EA}=10: 1)$ to give white needle-like crystals (PE:EA = 15:1). The yield was 60\%[11-12].

The yield was $60 \% .{ }^{1} \mathrm{H}$ NMR $\left(\mathrm{CDC1}_{3}\right): \delta(\mathrm{ppm})=3(\mathrm{t}, 2 \mathrm{H}, J=6.8 \mathrm{~Hz}), 4.37(\mathrm{t}$, $2 \mathrm{H}, J=6.8 \mathrm{~Hz}), 3.39(\mathrm{t}, 2 \mathrm{H}, J=6.4 \mathrm{~Hz}), 2.09(\mathrm{t}, 2 \mathrm{H}, J=7.2 \mathrm{~Hz}), 2.05(\mathrm{t}, 2 \mathrm{H}, J=6.8$ $\mathrm{Hz}){ }^{13} \mathrm{C} \mathrm{NMR}\left(\mathrm{CDC1}_{3}\right): \delta(\mathrm{ppm})=28.1,30.7,33.5,42.6,108.9,119.4,120.8,123.3$, 126.1, 140.7.

\section{Synthesis \\ of \\ 9,9}

-((sulfonylbis(4,1-phenylene))bis(oxy))bis(ethane-2,1-diyl))bis(9H-carbazole)

A $100 \mathrm{~mL}$ round bottom flask was charged with 4,4'-sulfonyl diphenol (100 mg), 9-(2-bromoethyl)-9H-carbazole (240 mg), and anhydrous $\mathrm{K}_{2} \mathrm{CO}_{3}(220 \mathrm{mg})$. A small amount of 18-crown-6 and anhydrous acetone $(40 \mathrm{~mL})$ were added under nitrogen. 
The mixture was heated at $60{ }^{\circ} \mathrm{C}$ with stirring for $48 \mathrm{~h}$. The reaction was stopped and the mixture was cooled to room temperature. The mixture was rinsed with acetone and the solvent was then removed. The resulting solid was dissolved in methylene chloride, $100 \mathrm{~mL}$ of distilled water was added, and the mixture was extracted with a separatory funnel. The organic layer was dried overnight, filtered, and the solvent was removed to give a pale yellow solid. The pale yellow solid was separated by column chromatography (PE:EA $=2: 1$ ) and a white solid was obtained.

The yield was $65 \% .{ }^{1} \mathrm{H}$ NMR $\left(\mathrm{CDC1}_{3}\right): \delta(\mathrm{ppm})=8.07(\mathrm{~d}, 2 \mathrm{H}, J=7.6 \mathrm{~Hz}), 7.69$ $(\mathrm{d}, 2 \mathrm{H}, J=8.8 \mathrm{~Hz}), 7.45(\mathrm{~d}, 4 \mathrm{H}, J=7.6 \mathrm{~Hz}), 7.25(\mathrm{~m}, 4 \mathrm{H}, J=7.2 \mathrm{~Hz}), 6.77(\mathrm{~d}, 2 \mathrm{H}, J$ $=8.4 \mathrm{~Hz}), 4.703(\mathrm{t}, 2 \mathrm{H}, J=5.6 \mathrm{~Hz}), 4.343(\mathrm{t}, 2 \mathrm{H}, J=5.6 \mathrm{~Hz}) ;{ }^{13} \mathrm{C} \mathrm{NMR}\left(\mathrm{CDC}_{3}\right): \delta$ $(\mathrm{ppm})=161.70,140.38,134.28,129.41,125.80,123.07,120.43,119.36,114.77$, 108.56, 66.36, 42.25; IR ( $\left.\mathrm{cm}^{-1}\right): 1621,1590.65,1492,1148,1152,1451,1324,1298,828$, 725.Elemental analysis: C, 75.45; H, 5.07; N, 4.40; O, 10.05; S, 5.03.

\section{Synthesis \\ of \\ 9,9}

-((sulfonylbis(4,1-phenylene))bis(oxy))bis(butane-4,1-diyl))bis(9H-carbazole)

A solution 4,4'-sulfonyl diphenol (100 mg) and 9-(4-bromobutyl)-9H-carbazole $\left(265.28 \mathrm{mg}\right.$ ) in a round bottom flask was charged with $225 \mathrm{mg}$ anhydrous $\mathrm{K}_{2} \mathrm{CO}_{3}$. A small amount of 18-crown-6 was added under magnetic stirring, and $40 \mathrm{~mL}$ of anhydrous acetone was added under vacuum and nitrogen. The mixture was heated at $60{ }^{\circ} \mathrm{C}$ with stirring reflux for $48 \mathrm{~h}$. The reaction was stopped and the mixture was cooled to room temperature. The mixture was then rinsed with acetone, the solvent was removed, and the resulting solid was dissolved in methylene chloride. To the solution, $100 \mathrm{~mL}$ of distilled water was added and the solution was extracted with a separatory funnel. The organic layer was dried, filtered, and the solvent was removed to give a pale yellow solid, which was separated by column chromatography (PE:EA $=2: 1$ ) and a white solid was obtained.

The yield was $65 \% .{ }^{1} \mathrm{H}$ NMR $\left(\mathrm{CDC}_{1}\right): \delta(\mathrm{ppm})=8.09(\mathrm{~d}, 2 \mathrm{H}, J=8 \mathrm{~Hz}), 7.79(\mathrm{~d}$, $2 \mathrm{H}, J=8.8 \mathrm{~Hz}), 7.42(\mathrm{~m}, 4 \mathrm{H}, J=7.2 \mathrm{~Hz}), 7.33(\mathrm{t}, 4 \mathrm{H}, J=8 \mathrm{~Hz}), 6.847(\mathrm{~d}, 2 \mathrm{H}, J=8.8$ $\mathrm{Hz}), 4.388$ (t, 2H, $J=6.8 \mathrm{~Hz}), 3.922(\mathrm{t}, 2 \mathrm{H}, J=6.0 \mathrm{~Hz}), 2.06(\mathrm{~m}, 2 \mathrm{H}, J=7.2 \mathrm{~Hz})$, $1.84(\mathrm{~m}, 2 \mathrm{H}, J=6.0 \mathrm{~Hz}) ;{ }^{13} \mathrm{C} \mathrm{NMR}\left(\mathrm{CDC1}_{3}\right): \delta(\mathrm{ppm})=162.34,140.32,133.94$, $129.49,125.68,122.90,120.43,118.93,114.75,108.54,67.88,42.61,26.80,25.69$; IR $\left(\mathrm{cm}^{-1}\right)=1621,1590.65,1492,1148,1152,1451,1324,1298,828,725$. Elemental analysis: C, 76.27; H, 5.82; N, 4.04; O, 9.24; S, 4.63 . 


\section{Results and Discussion}

\section{Photophysical properties of compound 3-1}

Figure 4a shows the UV spectra of compound 3-1 in different polar solvents. A whole field scan was performed and the concentration of 3-1 was $3 \times 10^{-5} \mathrm{~mol} \mathrm{~L}^{-1}$. From the figure, when the solvent polarity changes, the maximum absorption peak slight moves. However, the intensity is only slightly different in different solvents, indicating that under the conditions of the ground state the polarity of the solution has little effect on the intensity[14]. The compound has a strong absorption band between 290 and $300 \mathrm{~nm}$, indicating the $\pi-\pi^{*}$ transition absorption of the conjugated system. The fluorescence spectra of 3-1 under different polar conditions are shown in Figure $4 \mathrm{~b}$. The normalized fluorescence spectra of the compound in different polar solvents show a slight red shift with increasing polarity of the solvent[15-16], indicating that the polarity of the material under the conditions of the excited state has a significant effect on the normalized intensity. The reason for this phenomenon is that the interaction between the solute and the solvent causes the energy level of the highest occupied molecular orbital (HOMO) to move upwards so that the energy level of the lowest unoccupied molecular orbital (LUMO) decreases, the gap between the energy levels $\Delta E_{\mathrm{g}}$ decreases, and the emission wavelength red shifts.
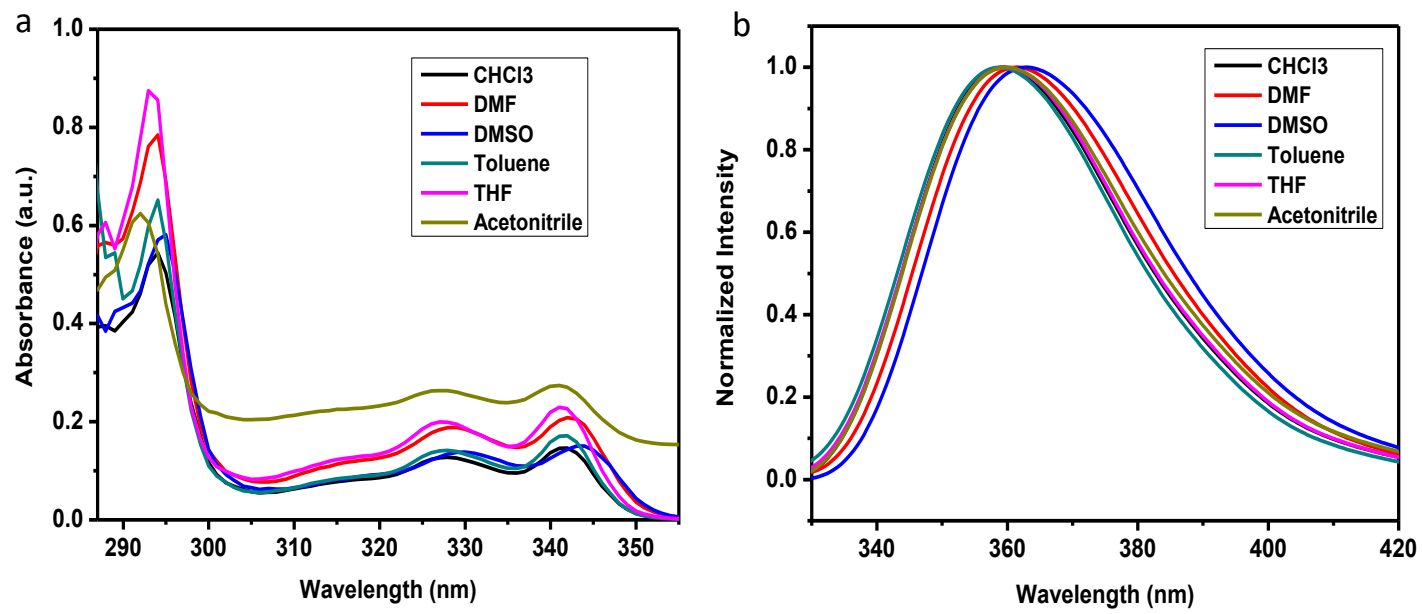

Fig. 4. a)UV absorption spectra of compound 3-1 in different solvents.b)Normalized fluorescence spectra of compound 3-1 in different solvents $\left(10^{-5} \mathrm{M}\right)$.

Figure 5 shows a comparison of the fluorescence spectra and UV absorption spectrum of the target compound in solution and the solid film. The wavelength of the compound shifts from 359.5 (solution) to $374.5 \mathrm{~nm}$ (solid) (a $15 \mathrm{~nm}$ red shift). Comparing the UV absorption spectrum and the fluorescence spectrum for the 
solution shows that a significant Stokes shift occurs. The maximum emission peak of the compound is between 359 and $375 \mathrm{~nm}(<400 \mathrm{~nm})$, so the material exhibits pure UV emission. The maximum emission peak in the solid state is also $<400 \mathrm{~nm}$. The external fluorescent quantum yields (QYs) of the compound 3-1 and 3-2 in the solid state are $0.66 \%$ and $4.81 \%$, respectively[23]. The compound has higher external quantum efficiency than other UV luminescent materials[24]. Compound 3-2 is a candidate for UV OLED materials.

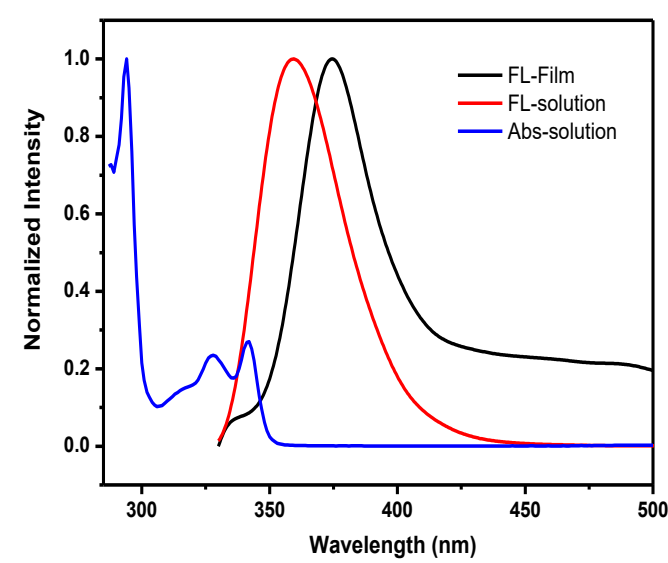

Fig. 5. Comparison of the photochemical properties of compound 3-1 in solution and in the solid state.

\section{Thermal properties}

The thermal stability of a material is important for device longevity. The thermograms of the compounds are shown in Figure 6. Thermogravimetric analysis reveals that the compounds have relatively high thermal stability. From the thermograms, the thermal decomposition temperatures of 3-1 and 3-2 are about 425 and $415{ }^{\circ} \mathrm{C}[21]$, respectively. This may be because with increasing alkyl chain length, distortion of the molecule increases and the molecular thermal stability decreases. These compounds have high overall thermal stability compared with other UV light-emitting materials[6,7].

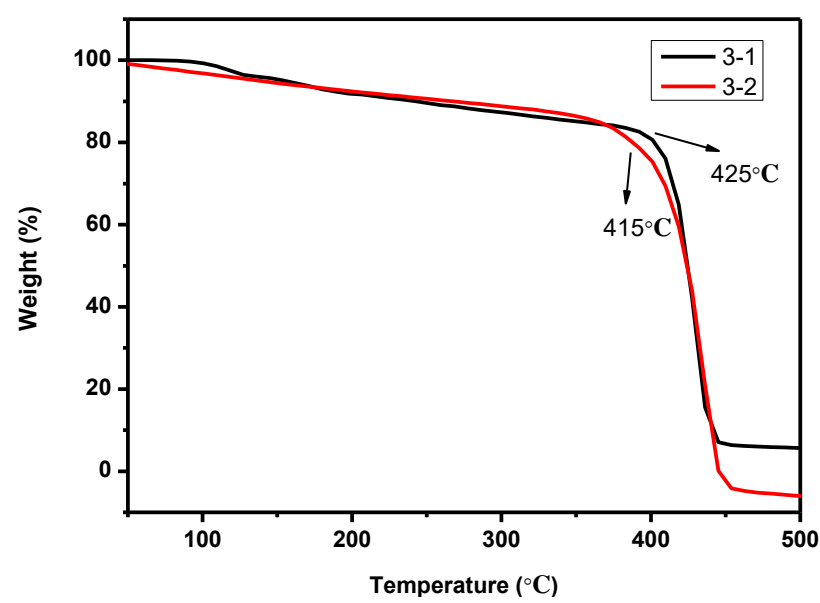


Fig. 6. Thermograms of compounds 3-1 and 3-2.

\section{Electrochemical properties}

The energy band gap $\Delta E_{\mathrm{g}}$ was calculated from the sideband of the UV spectrum using $\Delta E_{\mathrm{g}}=1240 / \lambda_{\text {abs. }}$. The sidebands of the UV absorption spectra of 3-1 and 3-2 are $357.03 \mathrm{~nm}$ and $360.04 \mathrm{~nm}$, so $\Delta E_{\mathrm{g}}$ are $3.47 \mathrm{eV}$ and $3.44 \mathrm{eV}$, respectively. From the electrochemical properties of the compounds, the compounds are quasi-reversible[17-18]. Compared with the commonly used cathode material Al, the energy level is $-4.3 \mathrm{eV}$, so the target compounds do not have a high electron transport barrier. Compared with the commonly used anode material indium tin oxide, the energy level is $-4.7 \mathrm{eV}$ and the HOMO level is $-5.33 \mathrm{eV}$, so there is not a high hole energy barrier[17-18] .From the electrochemical tests and calculations, the target compounds are expected to be OLED materials[20].

Table 2. Electrochemical properties of the target compounds.

\begin{tabular}{cccccccc}
\hline Compounds & $\lambda_{\text {oneset } / \mathrm{nm}}$ & Eoneset(OX)/ev & $\mathrm{EA} / \mathrm{ev}$ & $\mathrm{Lp} / \mathrm{ev}$ & $\Delta \mathrm{Eg} / \mathrm{ev}$ & $\mathrm{E}$ номо/ev & Elumo/ev \\
\hline $3-1$ & 357.03 & 0.57 & 1.837 & 5.31 & 3.473 & -5.31 & -1.837 \\
$3-2$ & 360.04 & 0.58 & 1.876 & 5.32 & 3.444 & -5.32 & -1.876 \\
\hline
\end{tabular}

\section{Theoretical calculations}

The HOMO and LUMO electron density distributions of 3-2 are shown in Fig. 7. The electron density of the HOMO is mainly distributed on the terminus of the carbazole group[22-23]. The electron density of the LUMO is mainly distributed on the sulfonamide group and its associated benzene ring structure. Structure optimization of the two compounds was performed by DFT at the B3LYP/6-31g level using Gaussian 09 software. The HOMOs and LUMOs of the compounds were visualized by Gauss View 5.0. For 3-2, the LUMO level is $-1.1309 \mathrm{eV}$ and the HOMO level is $-5.5984 \mathrm{eV}$, so the bandgap is $\Delta E_{\mathrm{g}}=E_{\mathrm{HOMO}}-E_{\mathrm{LUMO}}=4.4675 \mathrm{eV}$. From electrochemical cyclic voltammetry, the energy gap is $\Delta E_{\mathrm{g}}=3.44 \mathrm{eV}[24-27]$. The band gaps from electrochemical cyclic voltammetry and the theoretical calculations indicate that the compound has a wide band gap, which is required for an UV luminescent material so the compound is a candidate for UV OLEDs. 


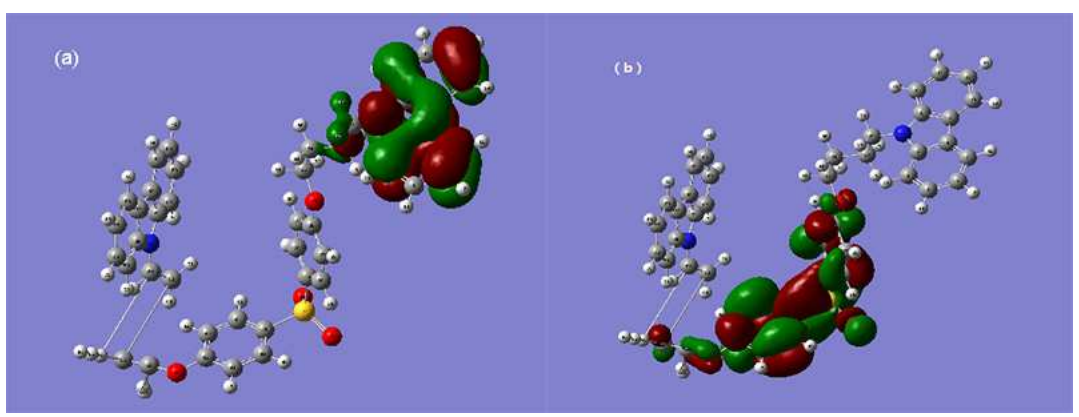

Fig .7. (a) HOMO and (b) LUMO electron density distributions of 3-2.

\section{Conclusions}

Two UV luminescent compounds have been designed and synthesized. The compounds have high thermal stability. The light-emitting regions of the compounds are in the long-wave UV region, so the compounds are expected to be good UV OLED materials[28-29]. The compounds are W-shaped and the crystals are layered structures. The $\mathrm{W}$ shape can reduce the $\pi-\pi$ interactions between molecules and reduce the fluorescence quenching effect. Thus, the molecules are candidates for UV OLED materials. The compounds have higher thermal stability than previously reported UV luminescent materials.

Funding This work was supported by the National Science Foundation of China (No. 21442004), the education office of Jilin Province (grant No. 2016320).

Authors' Contribution Experimental work done and Manuscript written by Huixian Jia, Manuscript checked and supervised by Chenghong Feng ,Wanfei Han, Yan Xue,Yanren Jin and Dongfeng Li.All authors read and approved the final manuscript.

Data Availability All data generated or analyzed during this study are included in this published article and its supplementary materials.

\section{Declarations}

Conflict of Interest The authors declared that there is no conflict of interest.

Ethical Approval Not applicable.

Consent to Participate Not applicable

Consent for Publication Not applicable

Code Availability Not applicable

\section{References}

1.Li G, Zhao J, Zhang D, et al. [J]. Journal of Materials Chemistry C, 2016, 4(37): 8787-8794.

2.Zhang Q, Li J, Shizu K, et al.[J]. Journal of the American Chemical Society, 2012, 134(36): 14706-14709.

3.Xu B, Wu H, Chen J, et al. [J]. Chemical Science, 2017. 
4. Li J, Yu Y, Luo L, et al.[J]. Tetrahedron Letters, 2016, 57(21): 2306-2310.

5.Bui D A, Kraiczek K G, Hauser P C. Molecular absorption measurements with an optical fibre coupled array of ultra-violet light-emitting diodes[J]. Analytica Chimica Acta, 2017, 986: 95-100.

6.Zhang Q, Zhang X, Wei B. Highly efficient ultraviolet organic light-emitting diodes and interface study using impedance spectroscopy[J]. Optik-International Journal for Light and Electron Optics, 2015, 126(18): 1595-1597.

7.Bae J, Kim Y H, Kim H Y, et al. Ultraviolet light stable and transparent sol-gel methyl siloxane hybrid material for UV light-emitting diode (UV LED) encapsulant[J]. ACS applied materials \& interfaces, 2015, 7(2): 1035-1039.

8.Taranekar P, Baba A, Fulghum T M, et al.[J]. Macromolecules, 2005, 38(9): 3679-3687.

9.Bogdal D, Jaskot K..[J]. Synthetic Communications, 2000, 30(18): 3341-3352.

10.Percec S, Tilford S. [J]. Journal of Polymer Science Part A: Polymer Chemistry, 2011, 49(2): 361-368.

11.Li L, Liu Y, Guo H, et al.[J]. Tetrahedron, 2010, 66(37): 7411-7417.

12.Bugatti V, Concilio S, Iannelli P, et al. [J]. Synthetic metals, 2006, 156(1): 13-20.

13.Sundararajan G, Rajaraman D, Srinivasan T, et al. Synthesis, characterization,computational calculation and biological studies of some2,6-diaryl-1-(prop-2-yn-1-yl) piperidin-4-one oxime derivatives[J]. Spectrochimica Acta Part A: Molecular and Biomolecular Spectroscopy, 2015, 139: $108-118$

14.Li R, Yan L, Wang Z, et al. An aggregation-induced emissive NIR luminescent based on ESIPT and TICT mechanisms and its application to the detection of Cys[J]. Journal of Molecular Structure, 2017, 1136: 1-6.

15.Li X, Mintz E A, Bu X R, et al. [J]. Tetrahedron, 2000, 56(32): 5785-5791.

16.Galievsky V A, Druzhinin S I, Demeter A, et al.[J]. The Journal of Physical Chemistry A, 2010, 114(48): 12622-12638.

17.Hou J, Pan Y, Jin J Y, et al. Isophorone-based analogues with A- $\pi$-D- $\pi$-A structure for red organic light emitting devices[J]. Synthetic Metals, 2009, 159(5): 401-405.

18.Wang $\mathrm{H}$, Liu $\mathrm{H}$, Bai $\mathrm{F}$ Q, et al. Theoretical and experimental study on intramolecular charge-transfer in symmetric bi-1,3,4-oxadiazole derivatives[J]. Journal of Photochemistry and Photobiology A: Chemistry, 2015, 312: 20-27. 
19.Pawluc P, Franczyk A, Walkow liak J,et al.[J]. Organic letters, 2011, 13(8): 1976-1979.

20 Paik K L, Baek N S, Kim H K, et al. White light-emitting diodes from novel silicon-based copolymers containing both electron-transport oxadiazole and hole-transport carbazole moieties in the main chain[J]. Macromolecules, 2002, 35(18): 6782-6791.

21 Yeh HC,Lee R H, Chan LH,et al.Synthesis,properties, and applications of tetraphenyl methane-based molecular materials for light-emitting devices[J]. Chemistry of materials, 2001, 13(9): 2788-2796.

22.Li D, Huang Z, Shang X, et al.[J]. Tetrahedron, 2015, 71(18): 2680-2685.

23.Zhang L, Xia Y, Li M, et al. [J]. Tetrahedron, 2016, 72(47): 7438-7442.

24.Lee J,Liu Q D,Motala M,et al.Photoluminescence,Electroluminescence, and Complex Formation of Novel N-7-Azaindolyl-and 2, 2-Dipyridylamino-Functionalized Siloles[J]. Chemistry of materials, 2004, 16(10): 1869-1877.

25.Ren X, Li J, Holmes R J, et al. Ultrahigh energy gap hosts in deep blue organic electrophosphorescent devices[J]. Chemistry of materials, 2004, 16(23): 4743-4747.

26.Liu X M, Lin T, Huang J, et al. Hyperbranched blue to red light-emitting polymers with tetraarylsilyl cores: Synthesis, optical and electroluminescence properties, and ab initio modeling studies[J]. Macromolecules, 2005, 38(10): 4157-4168.

27.Gibson H W, Brumfield K K, Grisle R A, et al. Synthesis of heterocyclic monomers via Reissert chemistry[J]. Journal of Polymer Science Part A: Polymer Chemistry, 2010, 48(17): 3856-3867.

28.A .Mikami.Y.Mizuno.S.Takeda.17.2:High Efficiency Ultraviolet Light Emitting Organic Devices and Its Application to White Light Sourcef .C.SID Symposium Digest of Technical Papers,2008, 39:215-218.

29.Zhang, Haiquan, et al. J.The Journal of Physical Chemistry B 108.28 (2004): 9571-9573.

\section{Graphical Abstract}




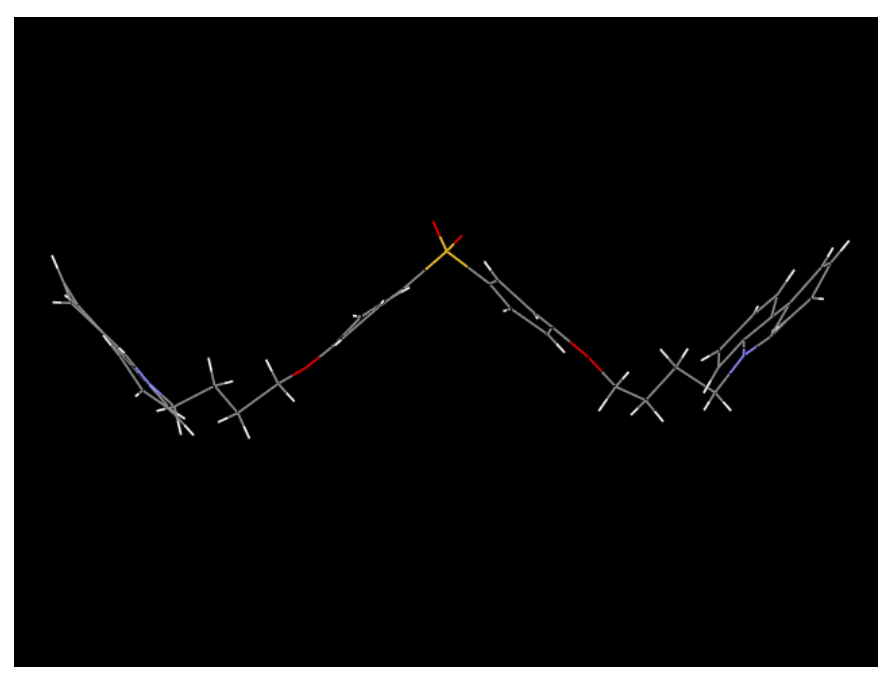




\title{
Supporting Information
}

\section{Synthesis and characterization of compounds based on carbazole and}

\section{sulfone groups}

\author{
Huixian Jia ${ }^{\mathrm{a}, \mathrm{b} *}$,Chenghong Feng ${ }^{\mathrm{c}}$,Wanfei Han ${ }^{\mathrm{a}}$, Yan Xue ${ }^{\mathrm{a}}$,Yanren Jin ${ }^{\mathrm{a}}$,Dongfeng $\mathrm{Li}^{\mathrm{b}}$
}

a Shanxi Xinhua Chemical Defense Equipment Research Institute Co., Ltd.., Taiyuan 030008, China

${ }^{b}$ School of Chemistry and Life Science, Changchun University of Technology, Changchun 130012, China

${ }^{c}$ State Key Laboratory of Water Environment Simulation, School of Environment, Beijing Normal University, Beijing 100875, China

*Corresponding authors Tel/Fax: +86 18435165116,

E-mailaddresses: huixian0401@163.com.;
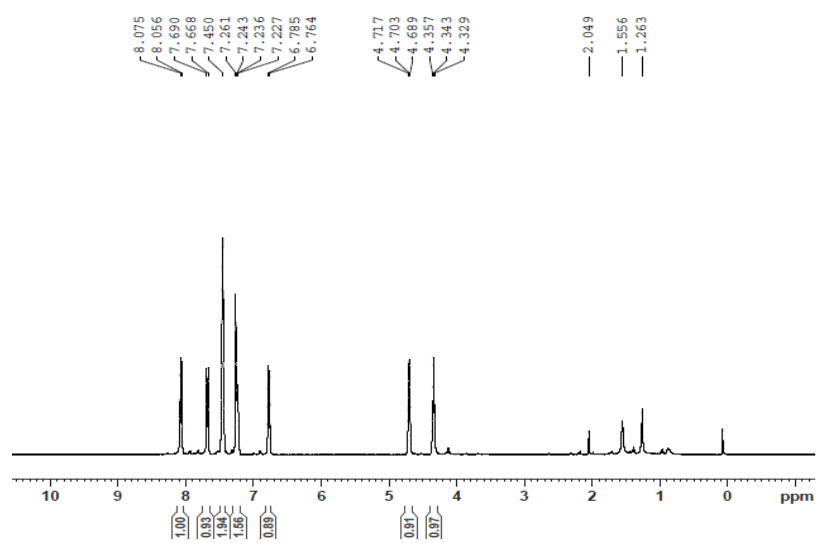

Fig. S1. ${ }^{1} \mathrm{H}$ NMR spectrum of compound 3-1.

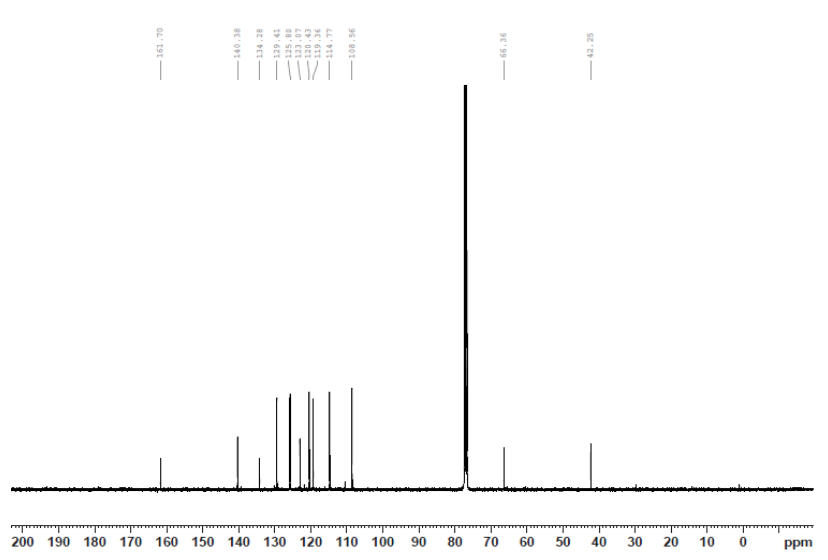

Fig. S2. ${ }^{13} \mathrm{C}$ NMR spectrum of compound 3-1. 


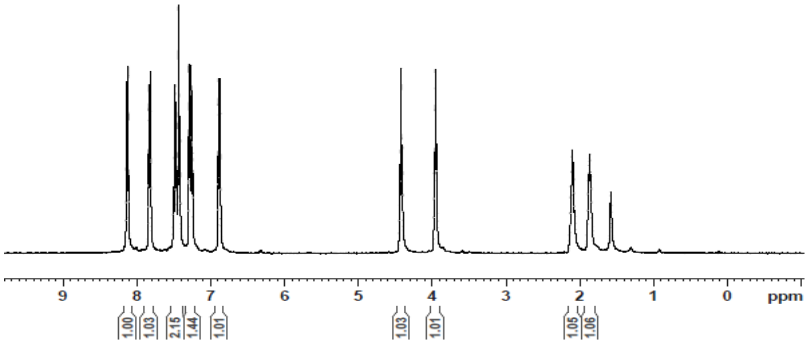

Fig. S3. ${ }^{1} \mathrm{H}$ NMR spectrum of compound 3-2.

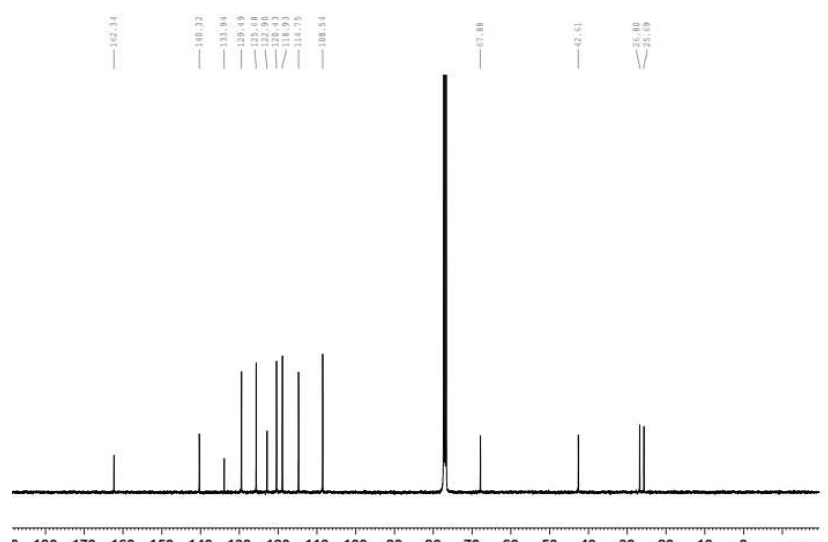

Fig. S4. ${ }^{13} \mathrm{C}$ NMR spectrum of compound 3-2.

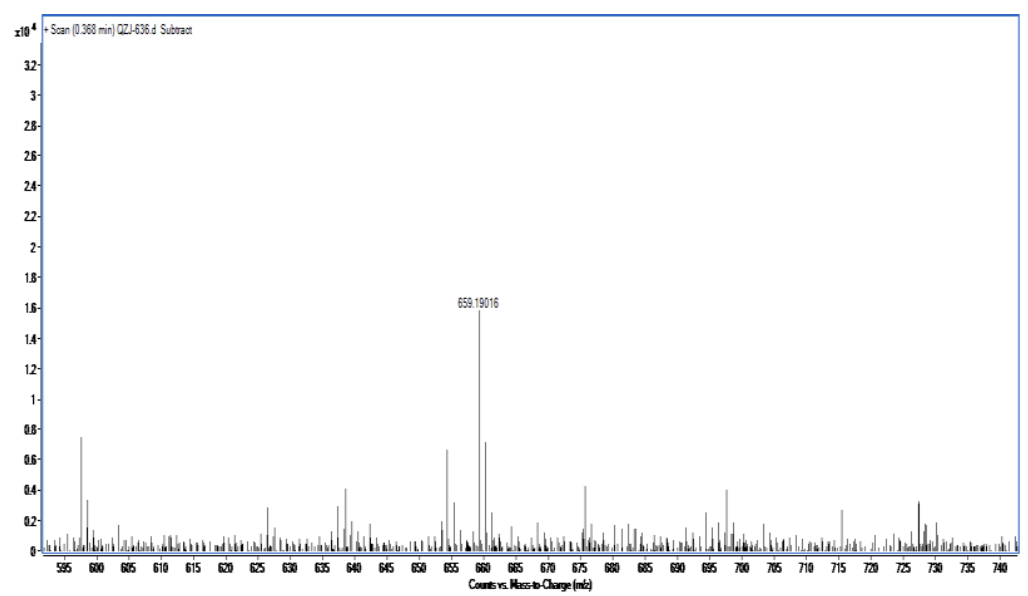

Fig. S5. Mass spectrum of compound 3-1. 


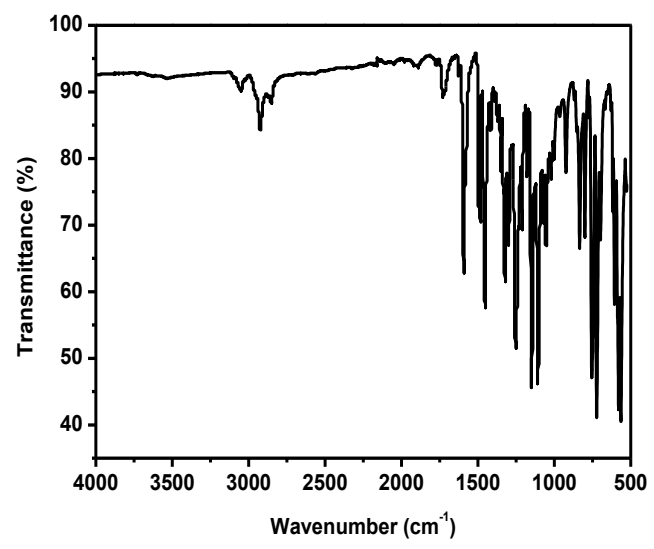

Fig. S6. FTIR spectrum of compound 3-1.

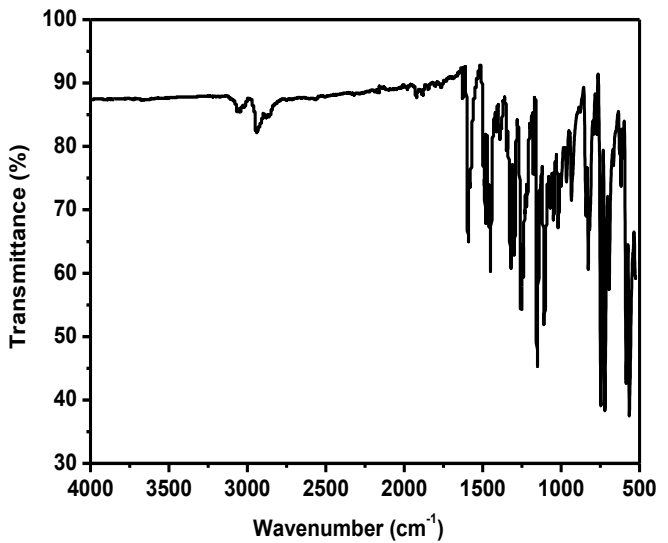

Fig. S7. FTIR spectrum of compound 3-2.

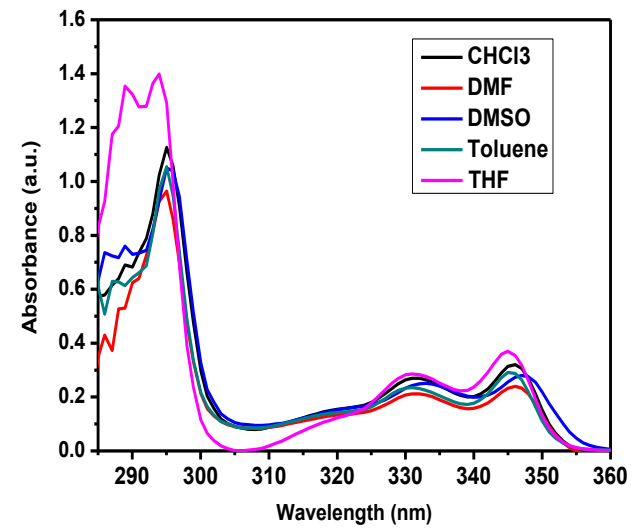

Fig. S8. UV absorption spectrum of compound 3-2 in different solvents $\left(10^{-5} \mathrm{M}\right)$. 


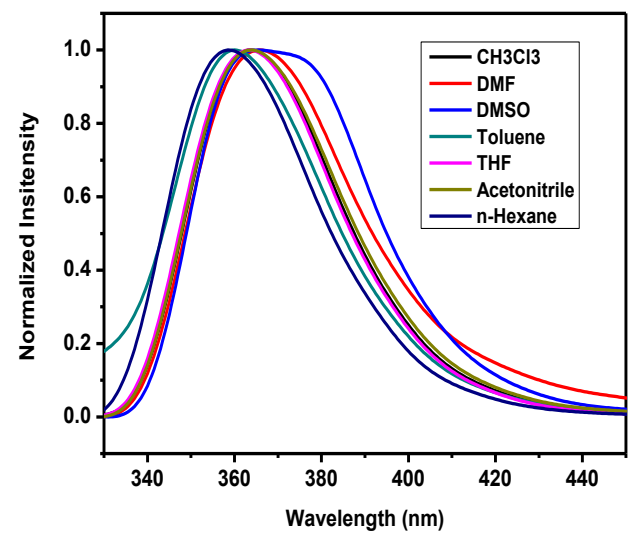

Fig. S9. Normalized fluorescence spectra of compound 3-2 in different solvents $\left(10^{-5} \mathrm{M}\right)$.

Table S1. 9-(4-Bromobutyl)-9H-carbazole crystal data.

\begin{tabular}{|c|c|}
\hline \multicolumn{2}{|l|}{ Crystal data } \\
\hline Chemical formula & $\mathrm{C}_{44} \mathrm{H}_{40} \mathrm{~N}_{2} \mathrm{O}_{4} \mathrm{~S}$ \\
\hline$M_{\mathrm{r}}$ & 692.27 \\
\hline Crystal system, space group & Orthorhombic, $\mathrm{Pbca}$ \\
\hline Temperature (K) & 273 \\
\hline$a, b, c(\AA)$ & $49.545(10), 4.9696(10), 14.321(3)$ \\
\hline$V\left(\AA^{3}\right)$ & $3520.4(12)$ \\
\hline$Z$ & 46 \\
\hline Radiation type & Mo $K \alpha$ \\
\hline $\mathrm{m}\left(\mathrm{mm}^{-1}\right)$ & 0.78 \\
\hline Crystal size (mm) & $0.30 \times 0.15 \times 0.15 \mathrm{~mm}$ \\
\hline \multicolumn{2}{|l|}{ Data collection } \\
\hline Diffractometer & $\begin{array}{l}\text { Bruker } \mathrm{P} 4 \\
\text { diffractometer }\end{array}$ \\
\hline Absorption correction & $\begin{array}{l}\text { Multi-scan } \\
S A D A B S 2014 / 5 \text { (Bruker,2014/5) was used } \\
\text { for absorption correction. wR2(int) was } \\
0.1658 \text { before and } 0.0900 \text { after correction. } \\
\text { The Ratio of minimum to maximum } \\
\text { transmission is } 0.3645 \text {. The } 1 / 2 \text { correction } \\
\text { factor is } 0.00150 \text {. }\end{array}$ \\
\hline$T_{\min }, T_{\max }$ & $0.275,0.754$ \\
\hline $\begin{array}{l}\text { No. of measured, independent and } \\
\text { observed }[I>2 \mathrm{~s}(I)] \text { reflections }\end{array}$ & $12283,7262,5475$ \\
\hline$R_{\text {int }}$ & 0.074 \\
\hline
\end{tabular}




\begin{tabular}{ll}
\hline$(\sin \mathrm{q} / \mathrm{l})_{\max }\left(\AA^{-1}\right)$ & 0.773 \\
Refinement & \\
$R\left[F^{2}>2 \mathrm{~s}\left(F^{2}\right)\right], w R\left(F^{2}\right), S$ & $0.546 \quad 0.942, \quad 5.79$ \\
No. of reflections & 2756 \\
No. of parameters & 7262 \\
H-atom treatment & H-atom parameters constrained \\
D $\rho_{\text {max }}, \mathrm{D} \rho_{\text {min }}\left(\mathrm{e} \AA^{-3}\right)$ & $29.99,-20.67$ \\
\hline
\end{tabular}

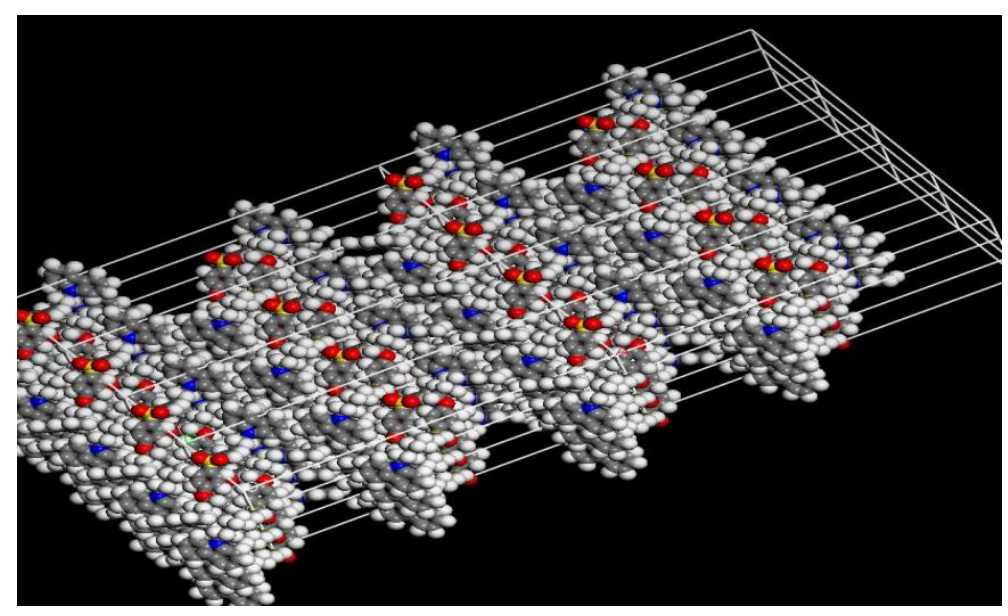

Fig. S10. Stacking of the target compound. 